\title{
Análise sobre a presença do cirurgião-dentista em Unidades de Terapia Intensiva da região metropolitana do Cariri-CE
}

\author{
Analysis of the presence of the dentist surgery in Intensive Care Units in \\ the metropolitan region of Cariri-CE \\ Análisis sobre la presencia del odontólogo en Unidades de Cuidados \\ Intensivos de la región metropolitana del Cariri-CE \\ Arthur Antônio Guedes GURGEL FILHO1 \\ Francisco Bernardo DUARTE NETO ${ }^{1}$ \\ Júlio Leite de ARAÚJO JÚNIOR ${ }^{2}$ \\ Italo Kennedy Silva SANTO ${ }^{3}$ \\ David Gomes de Alencar GONDIM ${ }^{4}$ \\ Ivo Cavalcante PITA NETO ${ }^{5}$ \\ Vilson Rocha Alencar CORTEZ 6 \\ Eduardo DIAS-RIBEIRO' \\ Francisco Aurelio Lucchesi SANDRINI ${ }^{8}$ \\ ${ }^{1}$ Graduado em Odontologia, Centro Universitário UNILEÃO, 63050-292 Juazeiro do Norte, CE, Brasil \\ ${ }^{2}$ Residente em Cirurgia e Traumatologia Bucomaxilofacial, Universidade Federal da Paraíba UFPB, 58051-790 João Pessoa - PB, Brasil \\ ${ }^{3}$ Graduando em Odontologia, Centro Universitário UNILEÃO, 63041-082 Juazeiro do Norte, CE, Brasil \\ ${ }^{4}$ Cirurgião e Traumatologista Bucomaxilofacial pela Universidade Federal do Pernambuco, UFPE \\ Professor de Cirurgia Bucal do Centro Universitário UNILEÃO, 63040-005 Juazeiro do Norte- CE, Brasil \\ ${ }^{5}$ Doutor em Ciências da Saúde pela Faculdade de Medicina do ABC Paulista, Santo André, \\ Cirurgião Dentista pela Universidade de Fortaleza UNIFOR, 63051-245 Juazeiro do Norte - CE, Brasil. \\ ${ }^{6}$ Cirurgião e Traumatologista Bucomaxilofacial pela Universidade Federal de Salvador, \\ Professor de Cirurgia Bucal da Centro Universitário UNILEÃO, 63040-000 Juazeiro do Norte - CE, Brasil. \\ ${ }^{7}$ Professor do Programa de Residência em Cirurgia e Traumatologia Bucomaxilofacial da UFPB, 58052-290 João Pessoa-PB, Brasil \\ ${ }^{8}$ Cirurgião e Traumatologista Buco-Maxilo-Facial pela Universidade Federal de Mato Grosso, \\ Professor de Cirurgia Bucal da Centro Universitário UNILEÃO, 63041-235 Juazeiro do Norte - CE, Brasil
}

\section{Resumo}

Introdução: A odontologia hospitalar enfrenta dificuldades que vão além do domínio profissional como o desafio do cirurgiãodentista sair de sua zona de conforto em consultórios até a necessidade de sua aceitação no âmbito hospitalar. OBJETIVOS: Analisar o conhecimento das equipes multidisciplinares/interdisciplinares que atuam em Unidades de Terapia intensivas da região metropolitana do Cariri-CE, sobre a participação e atuação do cirurgião dentista. Material e Método: A amostra foi composta de 40 profissionais atuante nas Unidades de Terapia intensivas da região metropolitana do Cariri-CE, avaliada através da aplicação de questionários estruturados a respeito do conhecimento sobre a atuação do cirurgião dentista na equipe. Resultados: 94,80\% acreditam que é importante a presença do cirurgião dentista nas em Unidades de Terapia intensivas. $61 \%$ dos profissionais citaram que sepse e infecções bucais podem ocorrer na maioria dos pacientes pela condição bucal precária; $11 \%$ citaram quadros de pneumonia, abcessos dentários e endocardite infeciosa. 58,8\% acreditam que o cirurgião dentista possa trazer redução dos custos hospitalares. Conclusão: Conclui-se que o entendimento da atuação do profissional de odontologia nas equipes multidisciplinares de em Unidades de Terapia intensivas, torna-se indispensável e imprescindível, para promoção e prevenção de agravos a saúde geral do paciente.

Descritores: Unidades de Terapia Intensiva; Endocardite; Doenças da Boca; Doenças da Gengiva.

\section{Abstract}

Introduction: Hospital dentistry faces difficulties that go beyond the professional domain as the challenge of the dental surgeon to leave his comfort zone in offices until the need for its acceptance in the hospital scope. Objectives: To analyze the knowledge of the multidisciplinary / interdisciplinary teams that work in intensive care units in the metropolitan region of Cariri-CE, about the participation and performance of the dentist surgeon. Methods: The sample consisted of 40 professionals working in the intensive care units of the metropolitan region of Cariri-CE, evaluated through the application of structured questionnaires regarding the knowledge about the performance of the dentist surgeon in the team. Results: $94.80 \%$ believe that the presence of the dental surgeon in the intensive care units is important. $61 \%$ of the professionals mentioned that sepsis and oral infections may occur in the majority of patients due to precarious oral conditions; $11 \%$ cited pneumonia, dental abscesses and infective endocarditis. $58.8 \%$ believe that the dental surgeon can reduce hospital costs. Conclusions: It is concluded that the understanding of the performance of the dentistry professional in the multidisciplinary teams of intensive care units is essential and essential for the promotion and prevention of injuries to the general health of the patient.

Descriptors: Intensive Care Units; Endocarditis; Mouth Diseases; Gingival Diseases.

\section{Resumen}

Introducción: La odontología hospitalaria enfrenta dificultades que van más allá del dominio profesional como el desafío del cirujano-dentista salir de su zona de confort en consultorios hasta la necesidad de su aceptación en el ámbito hospitalario. Objetivos: Analizar el conocimiento de los equipos multidisciplinares / interdisciplinarios que actúan en Unidades de Terapia intensivas de la región metropolitana del Cariri-Ce, sobre la participación y actuación del cirujano dentista. MÉTODOS: La muestra fue compuesta de 40 profesionales actuante en las Unidades de Terapia intensivas de la región metropolitana del Cariri-Ce, evaluada a través de la aplicación de cuestionarios estructurados acerca del conocimiento sobre la actuación del cirujano dentista en el equipo. Resultados: $94,80 \%$ creen que es importante la presencia del cirujano dentista en las Unidades de Terapia intensiva. El $61 \%$ de los profesionales citaron que la sepsis e infecciones bucales pueden ocurrir en la mayoría de los pacientes por la condición bucal precaria; $11 \%$ citaron cuadros de neumonía, abscesos dentales y endocarditis infeciosa. El 58,8\% cree que el cirujano dentista puede traer reducción de los costos hospitalarios. Conclusiones: Se concluye que el entendimiento de la actuación del profesional de odontología en los equipos multidisciplinarios de en Unidades de Terapia intensiva, se vuelve indispensable e imprescindible, para promoción y prevención de agravios a la salud general del paciente.

Descriptores: Unidades de Cuidados Intensivos; Endocarditis; Enfermedades de la Boca; Enfermedades de las Encías.

\section{INTRODUÇÃO}

O reconhecimento da prática odontológica no âmbito hospitalar ganhou ênfase na segunda metade do século XIX, a partir dos esforços dos Drs. Simon Hullihen e James Garrestson. Posteriormente, a Odontologia Hospitalar foi chancelada pela Associação Dental Americana, ganhando o respeito da classe médica ${ }^{1}$. Na maioria dos hospitais do Brasil, a presença do cirurgião-dentista na equipe multi/interdisciplinar da Unidade de Terapia Intensiva (UTI) 
é recente. Projeto de lei PL 2776/08, aguardando apreciação do Senado Federal, estabelece a obrigatoriedade do cirurgiãodentista na UTI no âmbito hospitalar da rede pública e privada $^{1-3}$

A avaliação das condições bucais em pacientes internados nas UTIs é relevante se considerarmos o expressivo comprometimento clínico. Submetidos a procedimentos altamente invasivos pela incapacidade de à incapacidade de nutrição, hidratação e respiração, esses pacientes apresentam alterações imunológicas importantes, além de desequilíbrio hídrico, o que concorre para a xerostomia, com consequente diminuição e espessamento da secreção salivar ${ }^{3}$.

De maneira geral, pacientes internados em Unidade de Terapia Intensiva não apresentam higiene bucal satisfatória. É importante que a Odontologia se integre ao atendimento dos pacientes hospitalizados nas UTIs, atuando na manutenção da higienização da cavidade bucal e controle de colonização intensa de patógenos, minimizando o risco de disseminação de microrganismos bucais que possam causar complicações sistêmicas ${ }^{4}$.

$\mathrm{O}$ presente estudo teve como objetivo avaliar o conhecimento de equipes multi/interdisciplinares que atuam em UTIs da região metropolitana do Cariri Cearense sobre a participação e atuação do cirurgião-dentista (CD) no âmbito hospitalar.

\section{MATERIAL E MÉTODO}

Foi realizado um estudo do tipo transversal qualitativo- quantitativo desenvolvido no período de outubro a novembro de 2016. Os participantes desta pesquisa foram profissionais que compõem as equipes multi/interdiciplinares das UTIs de dois Hospitais da Região Metropolitana do Cariri, (HRC e HSR). Foram incluídos na pesquisa médicos, técnicos de enfermagem, enfermeiros e fisioterapeutas atuantes nas UTIs e que concordaram em participar voluntariamente assinando o Termo de Consentimento Livre e Esclarecido (TCLE).

Após a submissão e aprovação do Comitê de Ética em Pesquisa do Instituto Leão Sampaio de Ensino Universitário Ltda., as informações foram coletadas por meio da aplicação de formulário contendo 22 questões, cujo objetivo foi avaliar o conhecimento da equipe multi/interdiciplinar sobre a atuação do CD em UTIs.

A pesquisa abrangeu todos os profissionais que compunham a equipe da UTI dos hospitais e que se dispuseram a participar da pesquisa, totalizando uma amostra de 40 profissionais. Após a coleta, os dados foram tabulados no programa Microsoft Excel 2010 e então foram desenvolvidas medidas de estatística descritiva a partir das medidas de tendencial central, valores absolutos e relativos, apresentando os resultados na forma de gráficos e tabelas.

\section{RESULTADOS E DISCUSSÃO}

Os dados obtidos foram apresentados em forma de tabelas e gráficos para a melhor visualização. A Tabela 1 apresenta a distribuição dos participantes em relação ao sexo e idade.

Observou-se que a maioria dos profissionais das UTIs relacionaram as alterações dos tecidos gengivais com infecções bucais e sepse. É sabido por $11 \%$ dos integrantes das UTIs que as patologias periodontais interferem na saúde geral do paciente, bem como podem gerar alterações em outros órgãos, Gráfico 1. Quando indagados sobre a necessidade de existência de programas de incentivo a participação do CD na UTI, foi observado uma maior porcentagem de aceitação desses profissionais, como visto no Gráfico 2.

Quando indagados sobre a necessidade de existência de programas de incentivo a participação do CD nas UTIs,
$75 \%$ responderam que deveria ocorrer sim, $17,5 \%$ declararam não saber e 7,50\% responderam que talvez devesse ocorre como visto no Gráfico 3.

Tabela 1. Distribuição do valor percentual das variáveis, sexo, idade em relação aos participantes na amostra $(n=40)$

\begin{tabular}{ccc}
\hline Variáveis & Categorias & \% \\
\hline \multirow{2}{*}{ Sexo } & Masculino & 22.5 \\
& Feminino & 77.5 \\
& $18-28$ & 25 \\
Idade & $29-39$ & 57.5 \\
& $40-50$ & 15 \\
& $>50$ & 2.5
\end{tabular}

*Fonte: dados da pesquisa

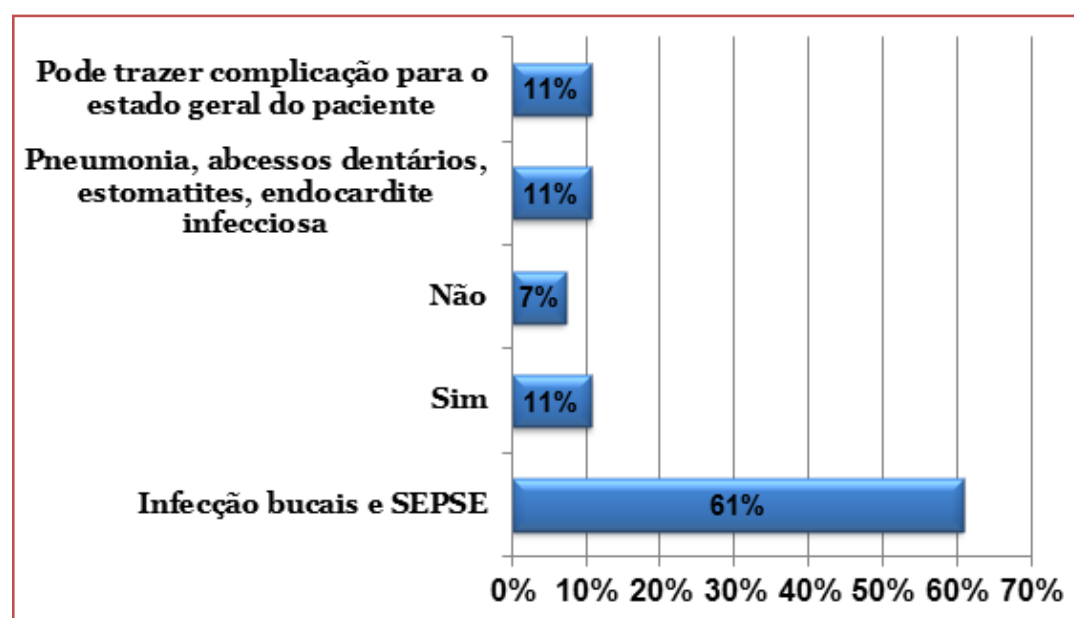

Gráfico 1: Respostas dos profissionais sobre os desfechos clínicos dos pacientes com alterações da saúde gengival (Fonte: dados da pesquisa).

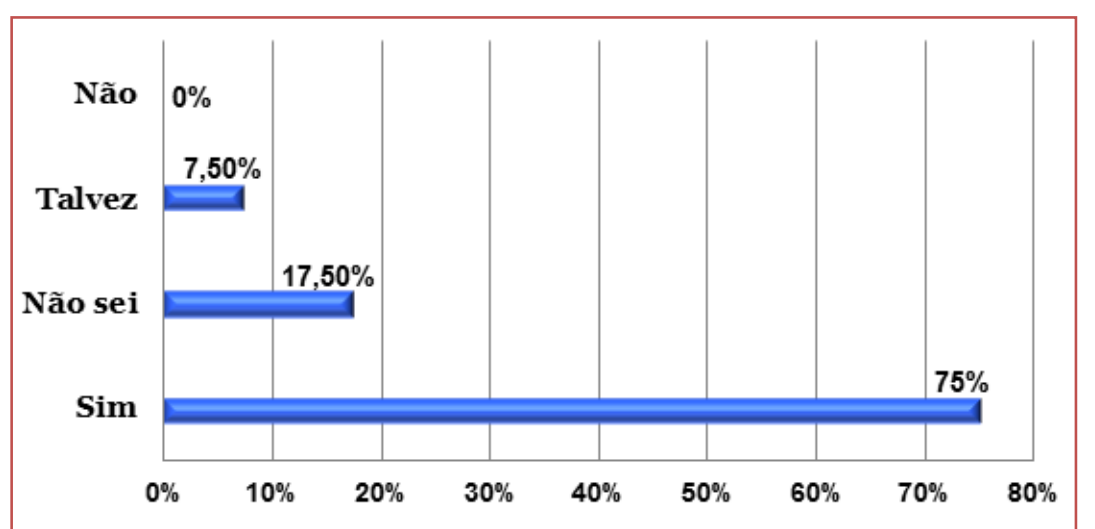

Gráfico 2: Opinião dos profissionais sobre a necessidade de existência de programas de incentivo a participação do CD na UTI (Fonte: dados da pesquisa)

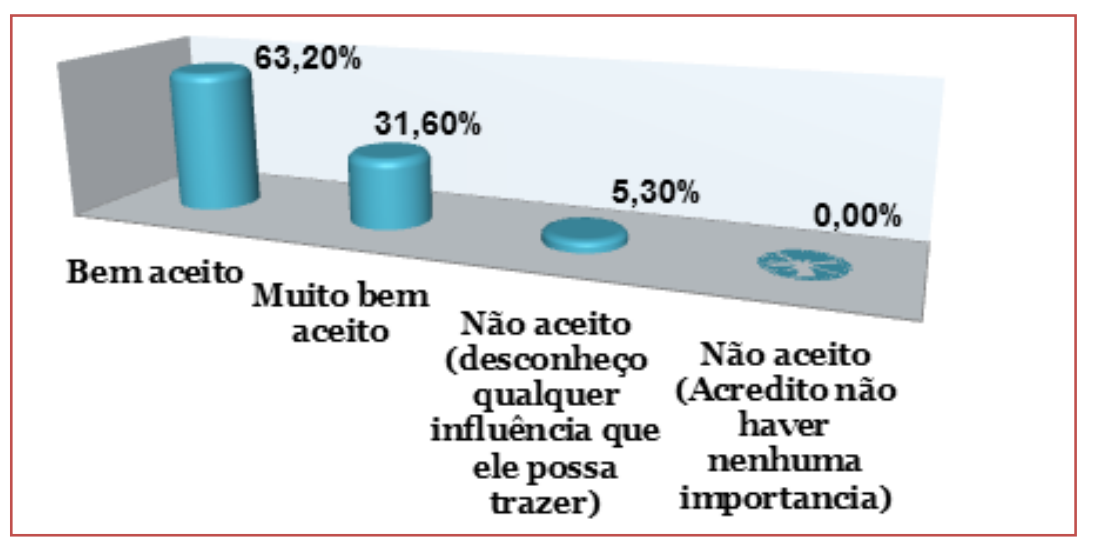

Gráfico 3: Valores percentuais de aceitação dos profissionais sobre a presença do CD na Unidade de Terapia Intensiva.

Sobre a aceitação dos profissionais quanto a inclusão do CD nas Unidades de Terapia Intensiva, 63,20\% avaliaram como bem aceito, $31,60 \%$ como muito bem aceito e 5,30\% assinalaram que não era aceito, pois desconhecia algum benefício do CD na UTI. Assim, observa-se uma ótima aceitação da inclusão do CD na UTI, entretanto, pequena parcela da amostra conhece bem o papel da Odontologia hospitalar.

Foi observado através da avaliação dos questionários, que os profissionais integrantes das UTIs estão cientes sobre a importância da inclusão do cirurgião-dentista na Unidade de Terapia Intensiva, bem como as principais implicações da 
condição bucal na saúde sistêmica dos internados. Com isso, é notória a inclusão do CD no âmbito hospitalar, uma vez que é o profissional com conhecimentos adequados para prevenir e tratar alterações na cavidade bucal.

Os integrantes das UTIs relataram a relação das infecções odontogênicas com sepse. Embora a sepse causada por infecção dentária seja rara, podem se disseminar rapidamente para os espaços profundos da face e pescoço e afetar outras estruturas, levando a complicações, como: comprometimento de vias aéreas, mediastinite, trombose de seio cavernoso ou até mesmo o óbito ${ }^{5,6}$

A odontologia hospitalar é uma área de suma importância para a saúde bucal e sistêmica dos pacientes que estão internados nos hospitais, proporcionando melhora no quadro clinico dos pacientes e consequentemente diminuem o tempo de internação. Também relatam que suas ações devem ser reconhecidas pela comunidade cientifica e pela população em geral ${ }^{6}$.

Os profissionais entrevistados acreditam que devem existir programas de incentivo e apoio a inclusão do Cirurgião-Dentista nas Unidades de Terapia Intensiva. O Projeto de Lei (PL) 2776/2008, já aprovado nas Comissões Especiais da Câmara dos Deputados e aguardando tramitação no Senado Federal, coloca a presença do $\mathrm{CD}$ não somente como traço da boa vontade dos gestores hospitalares, mas como elemento obrigatório da equipe multi/interdisciplinar assegurado por lei ${ }^{7}$.

O protocolo de higienização para melhora da saúde bucal dos pacientes internados na UTI é realizado pelos técnicos de enfermagem, os quais realizam aplicação de clorexidina a $0,12 \%$ quatro vezes ao $\mathrm{dia}^{8}$.

A literatura científica corrobora os dados levantados na pesquisa quanto à ótima aceitação do $\mathrm{CD}$ por outros profissionais, a despeito da pouca divulgação dos benefícios gerados pela presença do odontólogo na equipe. Certamente, paradigmas precisam ser substituídos, valorizando a atuação do CD nos hospitais cuja Unidade de Terapia Intensiva conte com a atuação desse profissional ${ }^{9,10}$.

\section{CONCLUSÃO}

Com base na metodologia empregada e nos resultados obtidos concluiu-se que a grande maioria dos profissionais das equipes multi/interdisciplinar mostrou-se consciente da importância da atuação do Cirurgião-Dentista nas Unidades de Terapia Intensiva, percebendo os benefícios que estes profissionais podem trazer à recuperação dos pacientes e aceitando de maneira positiva a divisão do espaço de trabalho.

Ademais, grande parte dos intensivistas reconhece as consequências sistêmicas das infecções bucais. Pode-se observar que a maioria dos profissionais acredita que a presença do $\mathrm{CD}$ traz melhorias na saúde do paciente internado, proporcionando uma diminuição no tempo de internação e reduzindo os custos nos serviços de saúde do âmbito hospitalar.

\section{REFERÊNCIAS}

1. Sousa LVS, Pereira AFV, Silva NBS. A atuação do cirurgião-dentista no atendimento hospitalar. Rev Ciênc Saúde. 2014; 16(1);39-45.

2. Silva ME, Resende VL, Abreu MH, Dayrell AV, Valle DDE A, Castilho LS. Oral hygiene protocols in intensive care units in a large Brazilian city. Am J Infect Control.2015; 43(3):303-4.

3. Amaral COF, Marques JA, Bovolato MC, Parizi AGS, Oliveira A, Straioto FG. Importância do cirurgiãodentista em Unidade de Terapia Intensiva: avaliação multidisciplinar. Rev Assoc Paul Cir Dent. 2013; 67(2):107-11.

4. Batista SA, Siqueira JSS, Silva Jr A, Ferreira MF, Agostini M, Torres SR. Alterações orais em pacientes internados em unidades de terapia intensiva. Rev Bras Odontol. 2014; 71(2):156-59.

5. Gonçalves PE, Rodrigues NALR, Seixas FL. Ações de promoção de saúde bucal no âmbito hospitalar. Rev Ciênc Méd. 2014; 23(1):15-23.

6. Silva AP, Caruso P, Jaguar GC, Carvalho PA, Alves FA. Oral evaluation and procedures performed by dentists in patients admitted to the intensive care unit of a cancer center. Support Care Cancer. 2014; 22:2645-50.

7. Gomes Filho IS, de Oliveira TS, da Cruz SS, PassosSoares Jde S, Trindade SC, Oliveira MT et al. Influence of periodontitis in the development of nosocomial pneumonia a case control study. J Periodontol. 2014; 85(5):e82-90.

8. Lima AKMMN, Cabral GMP, Araújo TLC, Franco MSP, Araújo Junior JL, Amaral RC. Percepção dos profissionais que trabalham na Unidade de Terapia Intensiva (UTI) quanto à inclusão do cirurgião-dentista na equipe. Full Dent Sci. 2016; 7(28):72-5.

9. Orlandini TRM, Basualdo A. Oliveira KC. Manutenção da higiene oral de pacientes internados em unidades de terapia intensiva de hospitais. J Oral Invest. 2013; 2(2):4-8

10. Araújo RJ, Oliveira LC, Hanna LM, Corrêa AM, Carvalho LH, Alvares NC. Perceptions and actions of oral care performed by nursing teams in intensive care units. Rev Bras Ter Intensiva. 2009;21(1):38-44.

\section{CONFLITO DE INTERESSES}

Os autores declaram não haver conflitos de interesse.

\section{AUTOR PARA CORRESPONDÊNCIA}

Júlio Leite de Araújo Júnior

juniorleitearaujo@hotmail.com

Submetido em 19/04/2018 Aceito em 30/05/2018 